\title{
Primary orbital precursor T-cell lymphoblastic lymphoma: Report of a unique case
}

\author{
LISA STENMAN $^{1}$, MARTA PERSSON ${ }^{2}$, FREDRIK ENLUND ${ }^{3}$, ERIK CLASEN-LINDE ${ }^{4}$, \\ GÖRAN STENMAN ${ }^{2}$ and STEFFEN HEEGAARD ${ }^{1,4}$
}

\author{
${ }^{1}$ Department of Ophthalmology, Rigshospitalet, University of Copenhagen, DK-2100 Copenhagen, Denmark; \\ ${ }^{2}$ Department of Pathology and Genetics, Sahlgrenska Cancer Center, University of Gothenburg, SE-40530 Gothenburg; \\ ${ }^{3}$ Department of Pathology and Genetics, Sahlgrenska University Hospital, SE-41345 Gothenburg, Sweden; \\ ${ }^{4}$ Department of Pathology, Rigshospitalet, University of Copenhagen, DK-2100 Copenhagen, Denmark
}

Received May 16, 2016; Accepted July 7, 2016

DOI: $10.3892 / \mathrm{mco} .2016 .1008$

\begin{abstract}
Primary T-cell lymphoblastic lymphoma (T-LBL) in the eye region is very rare. The present study described a unique case of T-LBL involving the extraocular muscles. A 22-year-old male patient presented with a 3 -week history of headache, reduced visual acuity and edema of the left eye. Clinical examination revealed left-sided exophthalmus, periorbital edema, chemosis, and reduced motility of the left eye. A magnetic resonance imaging scan revealed thickening of the left orbital muscles and a positron emission tomography-computed tomography scan also demonstrated activity in a subclavicular lymph node. Histopathological analysis of both lesions revealed infiltration by medium-sized neoplastic lymphoid cells with a high nuclear-cytoplasmic ratio and a high mitotic index. Immunostaining revealed positivity for CD2, CD3, CD99, Tia-1, and GranzymB, and variable positivity for CD4. There was no involvement of the bone marrow. Based on the clinical and histopathological findings, a diagnosis of T-LBL was made. There was no evidence of NOTCH1 mutation or rearrangements of the ETV6 and $M L L$ genes and high-resolution array-based comparative genomic hybridization (arrayCGH) analysis revealed a normal genomic profile. The patient received chemotherapy according to the high-risk NOPHO protocol, followed by myeloablative allogenic bone marrow transplantation. At 35 months after diagnosis, the patient remained in complete first remission, but without light perception on his left eye. To the best of our knowledge, this is the first report of a case of T-LBL involving the extraocular muscles. Although primary T-LBL in the eye region is very rare,
\end{abstract}

Correspondence to: Dr Steffen Heegaard, Department of Pathology, Rigshospitalet, University of Copenhagen, 11 Frederik V's Vej, DK-2100 Copenhagen, Denmark

E-mail: sthe@sund.ku.dk

Key words: T-cell lymphoblastic lymphoma, orbital manifestation, array-based comparative genomic hybridization analysis our findings demonstrate that lymphoma should be considered in the differential diagnosis of patients with similar symptoms.

\section{Introduction}

Lymphoblastic lymphoma (LBL) is a lymphoproliferative disorder composed of immature, neoplastic lymphocytes. T-cell LBL (T-LBL) is the second most common subtype of non-Hodgkin lymphoma in children and adolescents, comprising $\sim 85-90 \%$ of all LBLs $(1,2)$. It is most frequent among adolescent males, and is often characterized by rapid growth (1). T-LBL usually originates from lymph nodes or extranodal sites, such as the skin, liver, spleen, Waldeyer's ring, central nervous system and gonads. Approximately 50\% of the patients present with a mediastinal mass $(1,3)$. Up to $14 \%$ of extranodal non-Hodgkin lymphomas occur in the orbit (4). The eye region may also be secondarily affected by lymphomas arising in other extranodal sites, and 5\% of all non-Hodgkin lymphomas affect the eye region during the course of the disease (5).

Lymphoma is the most common orbital malignancy and comprises $\sim 10-20 \%$ of all orbital mass lesions. The majority of these cases are of B-cell lineage and the most common subtype is extranodal marginal B-cell lymphoma $(5,6)$. To the best of our knowledge, this is the first reported case of primary T-LBL developing in the orbit of a 22 -year-old man. The aim of this study was to present a detailed description of the clinical, histopathological, and genomic characteristics of this rare neoplasm.

\section{Case report}

Clinical history. An otherwise healthy 22-year-old man sought medical attention due to left-sided headache and swelling around the left eye for the past 3 weeks. Initially, the patient was administered antibiotics (Primcillin ${ }^{\circledR}$ ) by a general practitioner, due to a presumed sinusitis. The treatment was terminated as the symptoms worsened and the patient was subsequently referred to the Department of Ophthalmology at Rigshospitalet (Copenhagen, Denmark). The patient complained of a history of pain in the left eye, as well as fever and episodes of vomiting over the last few days, without other signs or history of systemic 
disease. Clinical examination revealed left-sided exophthalmus $(5 \mathrm{~mm})$, periorbital edema, chemosis, and reduced motility of the left eye (Fig. 1A). The visual acuity was $3 / 36$ in the left eye, with an ipsilateral positive relative afferent pupillary defect. Ophthalmoscopy revealed edema of the optic nerve head. A magnetic resonance imaging scan of the orbit revealed thickening of the medial and superior rectus muscle, as well as of the superior oblique muscle of the left eye (Fig. 1B). The right eye was unaffected. The blood profile revealed leukocytosis $12.5 \times 10^{9} / 1$ (ref. interval $\left.3.5-8.8 \times 10^{9} / 1\right)$ with neutrophilia $8.6 \times 10^{9} / 1$ (ref. interval $1.6-5.9 \times 10^{9} / 1$ ) and monocytosis $1.4 \times 19^{9} / 1$ (ref. interval 0.20-0.76x10\%/l). Hemoglobin and platelet count were within normal limits. Treatment with Prednisolon ${ }^{\circledR}$ was ineffective and 2 weeks later the patient experienced total loss of vision (no light perception) and complete loss of eye movements on the left side.

A positron emission tomography-computed tomography (PET-CT) scan also confirmed activity in an enlarged subclavicular lymph node. Biopsies of the orbital lesion and the enlarged subclavicular lymph node were performed, together with a bone marrow aspirate.

The patient started treatment according to the high-risk NOPHO protocol (7). High-dose chemotherapy, comprising cyclophosphamide, etoposide, intrathecal methotrexate, and cytarabine, led to reduced swelling of the left eye and left side of the face. The patient developed neutropenia and anemia, was hospitalized due to febrile neutropenia, and received several blood transfusions.

After the first three cycles of chemotherapy, a PET-CT scan revealed metabolic remission of the left orbit and eye, as well as of the subclavicular lymph node. The patient subsequently received three additional cycles of chemotherapy and two cycles of radiotherapy ( 2 Gy) followed by myeloablative allogenic bone marrow transplantation from an unrelated donor. At 35 months after diagnosis, the patient remained in complete first remission, but with no light perception in his left eye.

The study adheres to the tenets of the Declaration of Helsinki, and has been approved by the Local Scientific Ethics Committee (journal no. H-4-2013-003) and the Danish Data Protection Agency (journal no. 2012-41-0747). Written informed consent was obtained from the patient.

Histopathological and immunohistochemical findings. Microscopic examination of the orbital and subclavicular lymph node biopsy revealed infiltration by medium-sized neoplastic lymphoid cells, with a high nuclear-cytoplasmic ratio and a high mitotic index (Fig. 1C and D). Immunohistochemistry revealed positivity for CD2, CD3, CD99, Tia-1, and GranzymB (Fig. 1E and F) and variable positivity for CD4. Immunostaining for CD5, CD7, CD8, CD10, CD20, CD30, CD34, CD56, CD79 $\alpha$, ALK1, TdT, and $\mathrm{CD} 1 \alpha$ was negative. Based on the clinical and histopathological findings, a diagnosis of T-LBL was established.

Genomic profile. Conventional cytogenetic analysis of the bone marrow aspirate revealed a normal karyotype, without apparent chromosome rearrangements. Fluorescence in situ hybridization (FISH) analyses of bone marrow cells with probes for gene fusions involving $M L L(K M T 2 A), T C F 3-P B X 1$,
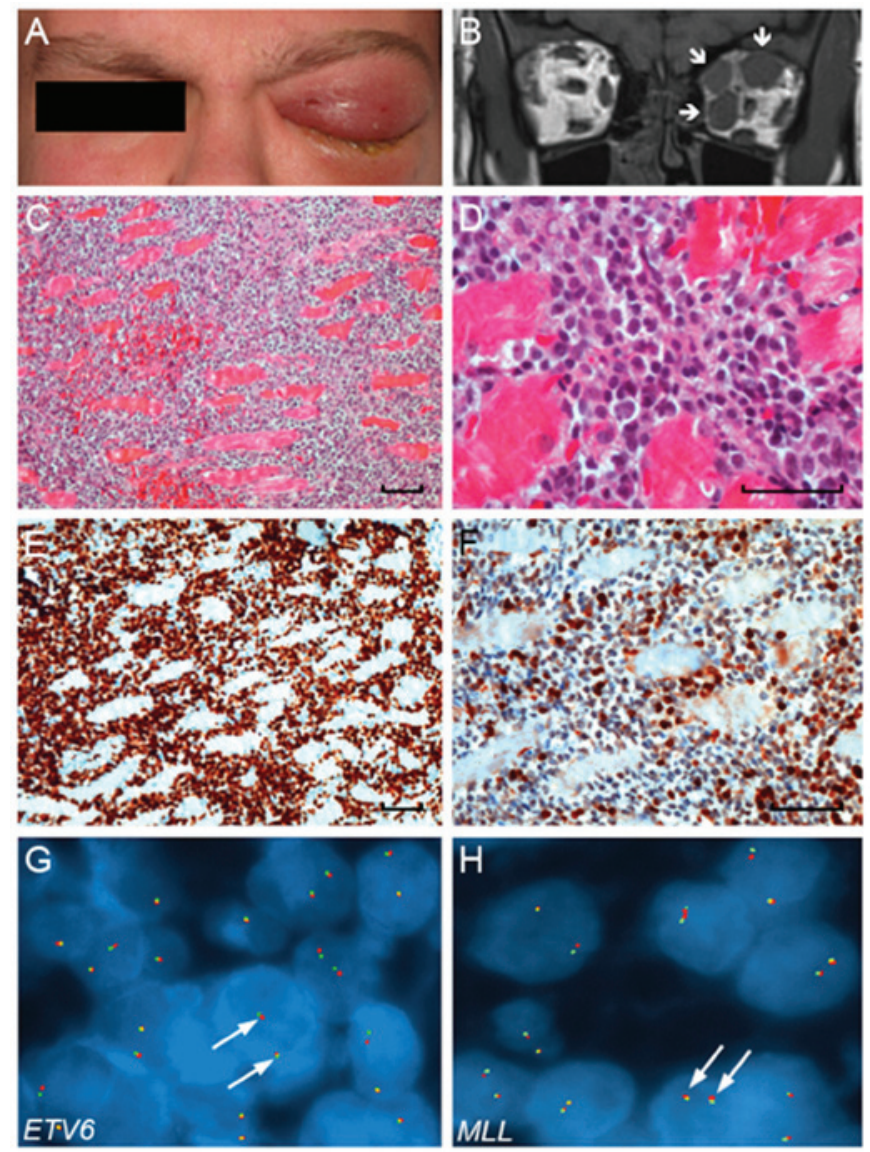

Figure 1. (A) A 22-year-old man presented with left-sided exophthalmos, discoloration of the skin and ptosis; (B) magnetic resonance imaging scan of both orbits (coronal view) demonstrating left-sided thickening of the medial and superior rectus muscles, and the superior oblique muscle (arrows); (C and D) pleomorphic tumor cells, with high nuclear-cytoplasmic ratio and scant cytoplasm, infiltrating the orbital muscles (hematoxylin and eosin staining; bar, $100 \mu \mathrm{m}$ ); the tumor cells displayed positive immunoreactivity for (E) CD3 and (F) CD99 (bar, $100 \mu \mathrm{m})$; (G and H) fluorescence in situ hybridization (FISH) analyses of formalin-fixed, paraffin-embedded tissue sections from the orbital biopsy revealed no rearrangements or translocations involving the ETV6 and MLL loci (two fused red and green signals/nuclei indicated by arrows).

BCR-ABL, and ETV6-RUNX1 (TEL-AML1), were also negative.

Array-based comparative genomic hybridization (arrayCGH) analysis of genomic DNA isolated from the orbital lesion (containing $>70 \%$ tumor cells) was performed with the Human Genome CGH Microarray 244K oligonucleotide array (G4411B; Agilent Technologies, Palo Alto, CA, USA). Data analysis was performed with Nexus Copy Number software v.7.5 (BioDiscovery Inc., El Segundo, CA, USA). Regions partially or completely covered by a previously reported copy number variation were excluded from the analysis $(8,9)$. ArrayCGH analysis of the orbital T-LBL revealed an apparently normal genomic profile. No major copy number alterations were detected and there was no evidence of homozygous deletions or gene amplifications.

To identify possible rearrangements of the ETV6 and $M L L(K M T 2 A)$ genes, we also performed FISH analysis of formalin-fixed, paraffin-embedded sections from the orbital lesion. The fluorescence signals were digitized, processed and analyzed with the Isis FISH imaging system v.5.5 
(MetaSystems GmbH, Altlussheim, Germany) and at least 50 nuclei were scored for each probe. Using dual-color FISH break-apart probes (Kreatech Diagnostics, Amsterdam, The Netherlands) we did not detect any evidence of rearrangements or copy number gain/amplifications of the ETV6 or MLL loci (Fig. $1 \mathrm{G}$ and $\mathrm{H}$ ).

Since NOTCH1 mutations in LBLs and leukemias are located predominantly within the heterodimerization domain and the C-terminal PEST domain, we sequenced exons 26-27, and 34 of the NOTCHI gene (10). However, no NOTCHI mutations were detected in any of the fragments sequenced ( $>70 \%$ neoplastic cells in the orbital biopsy from which the DNA was isolated).

\section{Discussion}

The present study presented a unique case of primary T-LBL involving the extraocular muscles of the left orbit of a 22-year-old previously healthy man. LBLs of the orbit are very rare, with only a few cases of $B-L B L$ reported in the literature $(5,6,11)$. To the best of our knowledge, this is the first case of T-LBL involving the orbit. Notably, a similar case of T-LBL, but affecting Tenon's capsule (fascia bulbi), was recently described in a 30-year-old man (4). Intramuscular, primary T-LBLs are exceedingly uncommon, with only one previously reported case of a 36-year-old male patient with intramuscular T-LBL involving the anterior, lateral, and deep posterior muscle compartments of the left leg (12). The symptoms of intramuscular LBLs are insidious and may include arthralgia, muscle swelling, and/or neuropathy (12). Our patient presented with proptosis, periorbital edema, pain, ptosis, restricted eye movements, and reduced visual acuity, which are symptoms typical of orbital lymphomas (3).

T-LBL and T-cell acute lymphoblastic leukemia (T-ALL) are considered to be the same disease, differing only by the extent of bone marrow infiltration $(1,6)$. Our patient exhibited no involvement of the bone marrow or peripheral blood. Lymphomas of the orbit are treated with moderate-dose orbital radiotherapy (3) and/or multiagent chemotherapy. In the Nordic countries, T-LBL and B-LBL are treated according to the NOPHO protocol, which is the same protocol as that used for lymphoblastic leukemias. Accordingly, our patient was also treated with the high-risk NOPHO protocol; he responded well and remains in complete first remission 35 months after the initiation of therapy. Using multiagent chemotherapy, the event-free survival rates for children and adolescents with T-LBL and B-LBL is $75-85 \%$ (2). By contrast, patients with relapsed disease have a poor outcome. Accurate diagnosis and prompt initiation of treatment according to high-risk protocols are therefore imperative for such patients.

To further characterize the present case of T-LBL, a high-resolution arrayCGH analysis was performed. The tumor had a stable genome, with no major detectable copy number alterations. Sequence analysis of frequently mutated regions of the NOTCH1 gene revealed no mutations. However, we cannot rule out the possibility of mutations located outside the hotspot regions sequenced. We were also unable to detect any rearrangements of the MLL and ETV6 genes. Further studies using whole exome and RNA sequencing may reveal novel oncogenic driver mutations in cases with a normal genomic profile. Epigenetic changes may also contribute to neoplastic transformation in such cases.

A previous study of pediatric patients with T-LBL has shown that mutations in NOTCHI (involved in the regulation of early T-cell development) are associated with a favorable prognosis (2). Similar observations have also been made in T-ALL (10). Conversely, patients with T-LBL and loss of heterozygosity of 6q14-24 have a poor prognosis and an increased risk of relapse (2).

T-LBL is an aggressive form of non-Hodgkin lymphoma (6). It is therefore important to consider T-LBL in the differential diagnosis of patients with symptoms such as proptosis, reduced visual acuity and mobility of the eye, periorbital edema, ptosis, fever, and pain. Prompt diagnosis and treatment of these patients are imperative in order to improve their prognosis and outcome.

\section{Acknowledgements}

This study was supported by the Synoptik-Fonden, the Swedish Cancer Society, and BioCARE, a National Strategic Cancer Research Program at the University of Gothenburg.

\section{References}

1. Jaffe ES, Harris NL, Stein H and Vardiman JW (eds): World Health Organization Classification of Tumors. Pathology and Genetics Tumours of Haematopoietic and Lymphoid Tissues. IARC Press, Lyon: pp111-117, 2001.

2. Bonn BR, Rohde M, Zimmermann M, Krieger D, Oschlies I, Niggli F, Wrobel G, Attarbaschi A, Escherich G, Klapper W, et al: Incidence and prognostic relevance of genetic variations in T-cell lymphoblastic lymphoma in childhood and adolescence. Blood 121: 3153-3160, 2013.

3. Nutting CM, Jenkins CD, Norton AJ, Cree I, Rose GE and Plowman PN: Primary orbital lymphoma. Hematol J 3: 14-16, 2002.

4. Wadhwani M, Verma D, Agarwal K, Prakash O and Shukla S: T-cell lymphoblastic lymphoma of Tenon's capsule: An unusual presentation. Int Ophtalmol 34: 639-642, 2014.

5. Ferry JA, Fung CY, Zukerberg L, Lucarelli MJ, Hasserjian RP, Preffer FI and Harris NL: Lymphoma of the ocular adnexa: A study of 353 cases. Am J Surg Pathol 31: 170-184, 2007.

6. Faridpooya K, Mulder MM, Merks JH, de Smet MD, Pals ST and Saeed P: Precursor B-lymphoblastic lymphoma of the orbit in a child: An unusual presentation of non-Hodgkin lymphoma. Orbit 25: 153-157, 2006 .

7. Lund B, Åsberg A,Heyman M,Kanerva J,Harila-Saari A,Hasle H, Söderhäll S, Jónsson ÓG, Lydersen S and Schmiegelow K; Nordic Society of Paediatric Haematology and Oncology: Risk factors for treatment related mortality in childhood acute lymphoblastic leukemia. Pediatr Blood Cancer 56: 551-559, 2011.

8. MacDonald JR, Ziman R, Yuen RK, Feuk L and Scherer SW: The database of genomic variants: A curated collection of structural variation in the human genome. Nucleic Acids Res 42 (Database Issue): D986-D992, 2014.

9. Iafrate AJ, Feuk L, Rivera MN, Listewnik ML, Donahoe PK, Qi Y, Scherer SW and Lee C: Detection of large-scale variation in the human genome. Nat Genet 36: 949-951, 2004.

10. Fogelstrand L, Staffas A, Wasslavik C, Sjögren H, Söderhäll S, Frost BM, Forestier E, Degerman S, Behrendtz M, Heldrup J, et al: Prognostic implications of mutations in NOTCH1 and FBXW7 in childhood T-ALL treated according to the NOPHO ALL-1992 and ALL-2000 protocols. Pediatr Blood Cancer 61: 424-430, 2014.

11. Shinkuma S, Natsuga K, Akiyama M, Saito A, Saito W, Ota S, Kondo T, Abe R, Kodama K and Shimizu H: Precursor B-cell lymphoblastic lymphoma presented with intraocular involvement and unusual skin manifestations. Ann Hematol 87: 677-679, 2008

12. Lim Z, Gupta S, Salisbury JR, Elias D, Venkatram NK, Mufti GJ and Pagliuca A: T-cell lymphoblastic lymphoma presenting as an intra-muscular mass. Br J Haematol 132: 537, 2006. 